\section{Immunotherapy interest drives IDO deals}

Big pharma continues to pile in to indoleamine 2,3-dioxygenase 1 (IDO1) inhibitors, with Roche signing its second deal in what has been a flurry of activity to gain access to the immunotherapy target. Roche paid US\$25 million upfront and committed up to $\$ 530$ million in milestones to develop Curadev's CRD1152, a preclinical-stage small-molecule dual inhibitor of IDO1 and tryptophan 2,3-dioxygenase (TDO). Last October, Roche subsidiary Genentech also licensed preclinical-stage NLG919 (now Phase I candidate RG6078) from NewLink Genetics for an upfront payment of $\$ 150$ million plus up to $\$ 1$ billion in milestones. Bristol-Myers Squibb, meanwhile, acquired Flexus Biosciences in February this year for $\$ 800$ million upfront plus up to $\$ 450$ million in milestones for access to the biotech's preclinical-stage IDO-selective, IDO and TDO dual, and TDO-selective inhibitors. And in December last year, Pfizer paid iTeos Therapeutics $€ 24$ million upfront plus undisclosed milestones for rights to preclinical-stage IDO1 and TDO2 inhibitors.

The deals are an extension of industry's focus on cancer immunotherapies. IDO1, which is constitutively expressed in many cancers, and TDO catalyze the degradation of the essential amino acid tryptophan. T cells are sensitive to tryptophan shortage, and the tumour overexpression of IDO1 and TDO acts to prevent the local proliferation of T cells into the tumour microenvironment, effectively helping cancers to hide from the immune system. By blocking the IDO1 and TDO enzymes, drug developers hope to restore the immune response.

Proof-of-concept data could start rolling in soon. Incyte has advanced its IDO1 inhibitor epacadostat into Phase II development for cervical and ovarian cancers. NewLink is also testing its IDO inhibitor indoximod, which it is not developing with Roche, in a Phase II breast cancer trial. Many oncologists anticipate that these two drug classes might work best, however, when combined with checkpoint inhibitors such as those that block cytotoxic T-lymphocyte antigen 4 (CTLA4) or programmed cell death protein 1 (PD1) (Nature Biotech. 33, 321-322. 2015; Nature Rev. Drug Discov. 14, 374-375, 2015).

Asher Mullard

\section{Cancer market hits $\$ 100$ billion}

Payers worldwide collectively spent US $\$ 100$ billion on oncology drugs last year, shows a new report from IMS Health. Oncology sales were up by $10.3 \%$ over a 1 -year period, and by $6.5 \%$ compounded annually over the past 5 years. The authors forecast continued growth in

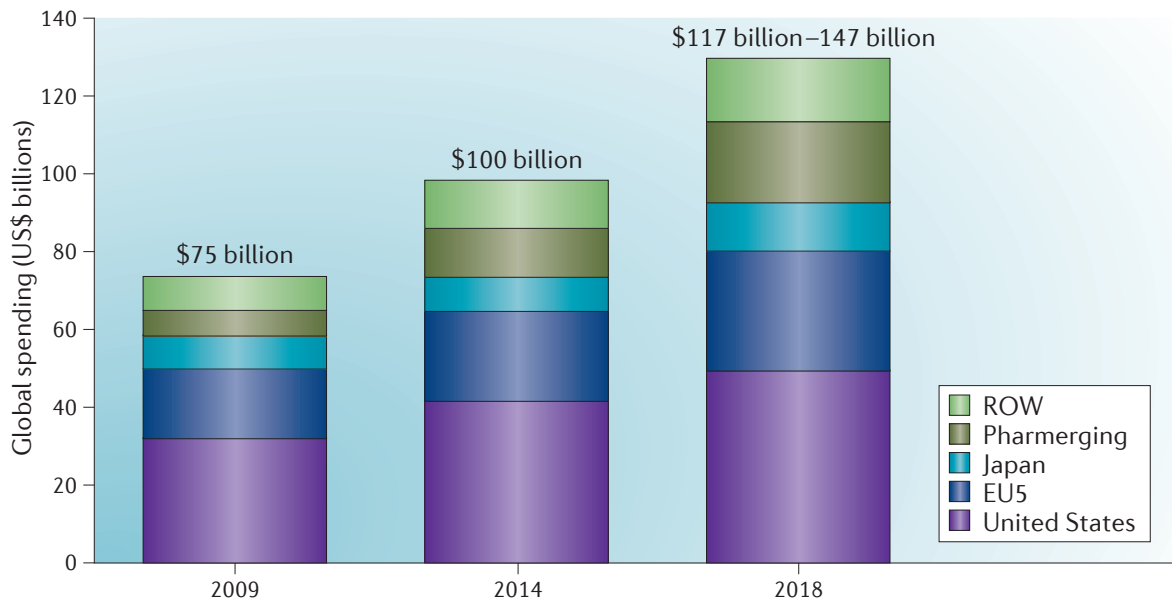

Figure 1 | Global oncology spending by year. EU5 markets are France, Germany, Italy, Spain and the United Kingdom. Pharmerging markets are Brazil, China, India, Mexico and Russia. ROW, rest of world. Sources: IMS Health MIDAS (December 2014) and IMS Health Market Prognosis (March 2015).
The report attributes the growth to earlier diagnosis of cancer, longer treatment duration and increased effectiveness of oncology drug therapies. But the authors caution that "measures of value continue to be tested by payers and providers who, in some health systems, most notably the US, have growing concerns about the financial burden faced by cancer patients".

Asher Mullard

\section{GSK scores first Phase III shingles trial success}

In a 15,411-patient Phase III trial, GlaxoSmithKline's Shingrix vaccine provided $97 \%$ protection against herpes zoster in patients aged 50 years and older, with a stable effect across all age groups (New Engl.). Med. 28 Apr 2015). This first of seven ongoing Phase III trials bodes well for the adjuvanted herpes zoster subunit vaccine. By contrast, Merck \& Co.'s approved live attenuated herpes zoster vaccine Zostavax reduced the incidence of herpes zoster by $70 \%$ in $50-59$-year-olds, by $64 \%$ in $60-69$-year-olds and by $38 \%$ in people aged 70 and older in its pivotal trials.

"The results for this vaccine are promising and may provide an important addition to vaccinations for an aging population," writes medical virologist Jeffrey Cohen, of the US National Institute of Allergy and Infectious Diseases, in an accompanying editorial (New Engl. J. Med. 28 Apr 2015). He adds that whereas Zostavax is contraindicated in people with impaired cellular immunity, who are at highest risk for herpes zoster, the subunit vaccine may provide a safe therapeutic option for these at-risk individuals. But, systemic adverse events were more than twofold more frequent in the Shingrix arm than in the placebo arm of the trial, providing some cause for pause. And Cohen noted that ongoing clinical trials still need to shed light on the duration of the effect of GSK's vaccine and on its ability to prevent post-herpetic neuralgia and other complications of herpes zoster in aged individuals.

Analysts expect GSK to file the vaccine for regulatory approval in the United States and in the European Union in 2016.

The vaccine uses the as-yet unapproved AS01B adjuvant, which is also a component of GSK's RTS,S malaria vaccine (also known as Mosquirix). RTS,S is currently under review with European regulators.

Asher Mullard 